\title{
Teardrop Glasses: Pseudo Tears Induce Sadness in You and Those Around You
}

\author{
Shigeo Yoshida \\ The University of Tokyo \\ Tokyo, Japan \\ JST, PRESTO \\ Tokyo, Japan \\ shigeodayo@cyber.t.u-tokyo.ac.jp
}

\author{
Takuji Narumi \\ The University of Tokyo \\ Tokyo, Japan \\ JST, PRESTO \\ Tokyo, Japan \\ narumi@cyber.t.u-tokyo.ac.jp
}

\author{
Tomohiro Tanikawa \\ The University of Tokyo \\ Tokyo, Japan \\ tani@cyber.t.u-tokyo.ac.jp
}

\author{
Hideaki Kuzuoka \\ The University of Tokyo \\ Tokyo, Japan \\ kuzuoka@cyber.t.u-tokyo.ac.jp
}

\begin{abstract}
Emotional contagion is a phenomenon in which one's emotions are transmitted among individuals unconsciously by observing others' emotional expressions. In this paper, we propose a method for mediating people's emotions by triggering emotional contagion through artificial bodily changes such as pseudo tears. We focused on shedding tears because of the link to several emotions besides sadness. In addition, it is expected that shedding tears would induce emotional contagion because it is observable by others. We designed an eyeglasses-style wearable device, Teardrop glasses, that release water drops near the wearer's eyes. The drops flow down the cheeks and emulate real tears. The study revealed that artificial crying with pseudo tears increased sadness among both wearers and those observing them. Moreover, artificial crying attenuated happiness and positive feelings in observers. Our findings show that actual bodily changes are not necessary for inducing emotional contagion as artificial bodily changes are also sufficient.
\end{abstract}

\section{CCS CONCEPTS}

- Human-centered computing $\rightarrow$ Interaction devices.

\section{KEYWORDS}

emotional contagion, emotion, wearable device, pseudo tears, artificial crying, embodied cognition

\section{ACM Reference Format:}

Shigeo Yoshida, Takuji Narumi, Tomohiro Tanikawa, Hideaki Kuzuoka, and Michitaka Hirose. 2021. Teardrop Glasses: Pseudo Tears Induce Sadness in You and Those Around You. In CHI Conference on Human Factors in Computing Systems (CHI '21), May 8-13, 2021, Yokohama, Japan. ACM, New York, NY, USA, 12 pages. https://doi.org/10.1145/3411764.3445741

This work is licensed under a Creative Commons

Attribution-NonCommercial-NoDerivs International 4.0 License.

CHI '21, May 8-13, 2021, Yokohama, Japan

(C) 2021 Copyright held by the owner/author(s)

ACM ISBN 978-1-4503-8096-6/21/05

https://doi.org/10.1145/3411764.3445741
Michitaka Hirose

The University of Tokyo Tokyo, Japan

hirose@cyber.t.u-tokyo.ac.jp

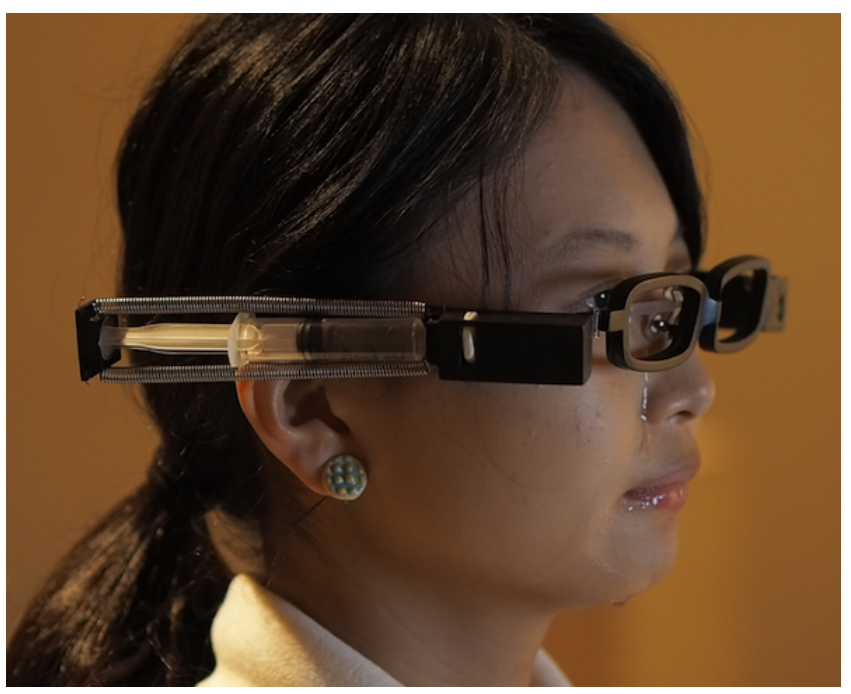

Figure 1: Teardrop glasses: An eyeglasses-style wearable device that aims to trigger emotional contagion with pseudo tears. A drop of water flows onto the face near the inner corners of the wearer's eyes, and the water flows down his/her cheeks.

\section{INTRODUCTION}

Feeling-as-information theory posits that emotions operate as information sources to signal what is happening in the surrounding environment, affecting our behaviors and decision-making unintentionally [57]. Although emotions play critical roles in everyday life, we do not necessarily know how we interact with our emotions. Therefore, our interest is in exploring technologies to mediate emotions to people and thereby uncover the interaction mechanism between our emotions and the environment.

Research in cognitive science have shown that a particular emotion can be elicited as a result of bodily changes [34]. According to the facial feedback hypothesis [58], an intentional smile enhances one's own posive feeling, but an intentional frown enhances the negative feeling. Interestingly, similar effects have been observed 
when simply holding a pen between one's lips or teeth [58] or by mimicking another person's facial expressions [39]. These facts show that an intentional change in expression leads to an unconscious change in one's emotion.

On the other hand, previous research have also found that the requirements for evoking emotions does not include only actual bodily changes but also one's recognition of those changes [56]. Some studies have revealed that actual bodily changes are not necessary for evoking emotions. For example, a study on false heart rate feedback [61] showed that the perceived attractiveness of an image can change when participants believe that the rapid or slow heartbeat heard is their own. The study showed that when artificial bodily changes are perceived as one's own, the perception changes one's emotion to match the perceived bodily changes.

Based on these studies, several researchers have been exploring interaction techniques that mediate intended emotions to users by making them believe that the artificially generated bodily changes are actually occurring in them [7, 8, 49, 62]. However, as one's own bodily changes are necessary to trigger one's emotions, those systems can only influence the direct users of those systems. This means that we have to prepare as many systems as the number of users. This would be a limitation when we intend to induce the emotions of multiple people simultaneously (e.g., amplifying emotions of people in a movie theater or regulating the emotions of people in a group therapy).

In contrast, a phenomenon in which emotion is unconsciously and instantly transmitted among people is known as emotional contagion. This is caused by observing another person's bodily changes [10, 27, 29] or other emotional expressions, which are physical manifestations of one's emotions [13, 22, 23, 41, 48]. We believe this phenomenon can be used to create an efficient system that can cause a chain reaction involving multiple people with a single device by simply presenting the bodily changes in one person.

In this study, we assumed that we can induce specific emotions in the person from whom the artificial bodily change is applied and the people around him/her observing the bodily changes and the accompanying emotional expressions. In particular, we focused on the shedding of tears as a trigger to induce certain emotions because crying is known to relate to various emotional states such as anger, happiness, joy, and sadness [20, 25, 44, 53]. Crying for emotional release is also known to be quite beneficial for stress relief and mental health $[3,6,19]$. Moreover, shedding tears is a bodily change observable by others, and visible tears promote empathy [30]. Then, we developed Teardrop glasses, which reproduce a sensation similar to that of the user crying (Figure 1). Drops of water fall near the inner corners of the user's eyes and flow down his/her cheeks. We believe that we can mediate emotions by allowing people to believe that the person presenting the artificial bodily change is actually shedding tears (i.e., artificial crying).

Our research makes the following contributions that can lead to a greater understanding of whether artificial bodily changes influence emotions:

(1) A novel concept that aims to mediate people's emotions through emotional contagion using artificial bodily changes.

(2) The design and development of a wearable device that aims to trigger emotional contagion with artificial bodily changes, such as pseudo tears, which are observable by surrounding people.

(3) The results of our user study show that both the individual expressing artificial crying with pseudo tears (wearer) and people who observed him/her (non-wearers) felt sad. Thus, pseudo tears can trigger emotional contagion.

(4) Moreover, we found that non-wearers are significantly more affected by the artificial crying than wearers of the device.

\section{BACKGROUND}

To build upon our research, we drew on psychological research investigating how bodily changes and emotions relate to each other and HCI research based on those findings. In addition, we reviewed relevant works on tears and emotions.

\subsection{Theories of Emotions}

2.1.1 Emotions and Bodily Changes. The mechanism of how we feel emotions has been studied for a long time, and various theories have been proposed and discussed throughout history. These theories of emotions share a similar notion that emotions are triggered by recognizing changes in one's own body. The James-Lange theory [35] states that involuntary bodily changes, such as heart rate, sweating, and muscle movements, caused by responses to specific stimuli, activate subjective feelings. In other words, we are sad because we cry, and we are happy because we smile. The two-factor theory of emotion [56] also supports such a paradoxical relationship between emotions and the body and states that bodily changes precede the experience of emotions, and these bodily changes influence our emotions. In addition, Damasio et al. argue that bodily changes occur prior to the experience of emotions and that emotions contribute to biasing decision-making [9].

Some studies have demonstrated that not only actual bodily changes but also artificial ones can trigger subjective feelings in people $[43,45,61]$. For example, Valins taped fast and slow heartbeat sounds and presented them as the participants' own. Interestingly, this manipulation caused the participants to alter their preferences/judgments [61]. Similar to our work, Mori et al. [45] focused on tearing and found that pseudo tearing on a participant's face caused him/her to feel sad. Liang et al. [43] reported that pseudo tears increase negative emotions and recall negative events.

2.1.2 Emotional Contagion. It is known that the emotions of others unconsciously influence ours. This phenomenon is called emotional contagion, in which we feel the same emotions as others when we perceive their bodily changes and emotional expressions. For example, you might feel sad when you see someone crying, happy when you hear a cheerful voice, and irritated when you are next to someone shaking his/her legs.

Emotional contagion involves the process of automatically imitating the bodily changes and emotional expressions of others, such as facial expression, voice tone, posture, and body movements [27]. The emotions are then triggered by the recognition of imitated bodily changes, as described above.

Research have also shown that emotions can be shared via online communications, such as a text chat $[22,23]$ and a social networking service $[13,40,41]$. The results showed that even observing 
emotional expressions without embodiment can influence our emotions.

In our study, unlike the above studies that investigated the direct effect of pseudo tears, we focused on investigating whether emotional contagion can be triggered by pseudo tears reproduced by our device.

\subsection{Attempts to Mediate Emotions in HCI}

Based on the theoretical background on emotions, some research have demonstrated the capability to enhance positive feelings [ 60 , 62], regulate negative feelings [7, 8], support the use of cognitive abilities [47, 59], augment entertainment/cinematic experiences $[16,18]$, and so on.

For example, Nishimura et al. [49] presented a tactile device for simulating a heartbeat and found the same results as those in Valins' work. Costa et al. [7] also focused on false heart rate feedback and developed a wearable device that aims to regulate a user's anxiety by providing a pseudo heartbeat vibration. Yoshida et al. [62] focused on pseudo facial expressions and created a mirrorlike system that reflects the user's facial expression as a slight smile or frown to induce positive and negative affects in the user. While these studies aimed to induce specific emotions in a person who is directly presented with an artificial bodily change, our work focuses on the mechanism of emotional contagion to mediate the emotional experiences of multiple people at once.

Some researchers have demonstrated that artificial bodily changes in others influence subjective feelings. Nakazato et al confirmed that creativity during a brainstorming session can be enhanced by slightly changing their facial expression and making them smile [47]. Suzuki et al. developed a video chat system in which his/her partner's facial expression is modified to synchronize with his/her own change in facial expression. They confirmed that the mirror effect induced by the system improved the impression of the partner during the conversation [59]. These studies showed that artificial bodily changes can be used to enhance our emotions and cognitive abilities, but primarily focused on enhancing an individual's emotions in response to the individual's voluntary emotional expression during communications. In addition, our study attempts to examine whether particular emotions can be mediated to multiple people via technologies.

On the other hand, Picard proposed the concept of Affective Computing [51] by relating emotions to computing. Based on this concept, many have studied methods to analyze and sense emotions [52], training systems [32], and interaction techniques [4] to better improve the human emotional experience with technology. In addition to Picard and her colleagues, researchers in HCI have also proposed interfaces that mediate emotions through haptics $[50,55]$, taste $[17,36]$, and other modalities [24, 33]. Our research is similar to affective computing, but our primary interest is mediating emotions based on the theories of emotions and bodily changes.

\subsection{Tears and Emotions}

Previous research have found that there is a relationship between emotional tears and a variety of emotions, such as sadness, happiness, anger, and joy [20, 25, 45, 53]. These emotional variations indicate that the emotion elicited by tears is context-dependent.
For example, people who were observed as crying while watching a sad movie were found to feel sadder than those who did not cry [20]. Furthermore, it was reported that the addition of tears to a neutral facial expression (digitally added using Photoshop) could increase the perceived need for support [2]. In contrast, participants remembering their most recent crying episode tended to feel positive rather than negative $[3,6]$. However, this was attributed to the emotional support received from people around the crying person whose emotions were recovered during his/her crying episode. Therefore, these studies indicate that, for tears to induce feelings other than sadness, the context of one's crying and the memory of social interactions during one's crying is necessary. Overall, these studies indicate that tears typically connote negative feelings, especially sadness, in the absence of social interactions with others and specific emotional contexts.

\section{DESIGN AND IMPLEMENTATION}

We developed a wearable device similar to eyeglasses called Teardrop glasses. This device places water drops near the inner corners of the user's eyes, but not in the eyes owing to hygiene considerations. The water subsequently rolls down the wearer's cheeks.

\subsection{Design Considerations}

Before designing the device, we considered the following issues that might influence the experience.

3.1.1 Components of Tears. Non-emotional tears consist of $98 \%$ to $99 \%$ water and the remaining is a protein substance [12]. Emotional tears, which are shed when a person's emotions change, have a higher concentration of protein than, for example, irritantinduced tears have by $24 \%$ [14]. However, the relationship between physiological effects and the compositions of tears remains unclear because the amount of tears that people produce is limited [46]. Nonetheless, the primary component of emotional tears is water, and it is conceivable that the proportions of water and protein in reproduced tears do not influence emotion evocation. Therefore, we used ordinary purified water that is harmless to humans to produce pseudo tears.

3.1.2 Location of Overflowing Tears. Tears are secreted by the lachrymal glands, which are located at the top of the outer corner of the eyes, and redundant tears flow into the puncta and the lachrymal duct, which are located at the inner corners of the eyes, caused by muscular actions during blinking $[1,63]$. In accordance with the flow of tears as described here, but depending on the shape of the eyes, the points at which tears overflow the lower eyelids tend to be near the inner corners of the eyes. Mori et al. described dropping water near the lachrymal ducts to invoke sadness [45]. Therefore, we selected the inner corners of the participants' eyes as the locations at which to apply drops of water as pseudo tears.

\subsection{Hardware Design}

We then designed an eyeglasses style wearable device to release a drop of water near the inner corners of the wearer's eyes. The frame of our device was 3D-printed and fabricated with UV-curable resin. The device consists of a battery (AAA), a control circuit, 

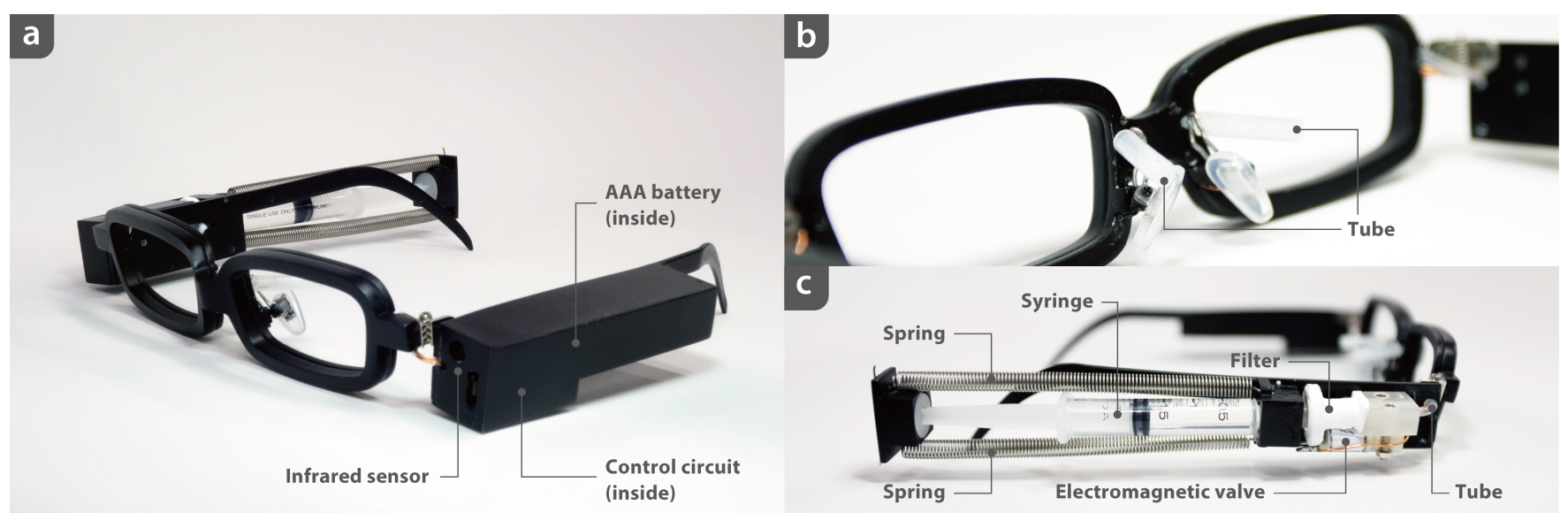

Figure 2: External view of Teardrop glasses. (a) An infrared sensor is positioned at the top of the left arm for communication between the control circuit and the communication module. The control circuit and aAA battery are inside the stems of the glasses. (b) Two tubes are attached around the areas of the inner corners of eyes. (c) A tube before splitting into two, a filter, and a syringe are connected to an electromagnetic valve.

an electromagnetic valve (LEE Company LHLA0352311H), two springs, a syringe with purified water, and tubes to carry water (Figure 2). The circuit board consists of an Arduino-compatible microcontroller (ATtiny85, 8 bit, $8 \mathrm{MHz}$ ), an infrared sensor, and a motor driver. The total weight of the device was $72 \mathrm{~g}$, including the water in the syringe. Its dimensions are approximately $175 \mathrm{~mm} \times$ $150 \mathrm{~mm} \times 40 \mathrm{~mm}$.

\subsection{Mechanism for Dropping Tear}

Water flows down in drops from two tubes that are located near the inner corners of the user's eyes (Figure 2 (b)). The position from which the water drops can be adjusted by changing the lengths of the tubes and the positions of the nose pads, by considering the variations in eye distance and size.

A syringe is used to store and refill the water and can be detached from the device (Figure 2 (c)). The syringe is attached to the electromagnetic valve via a small water filtration filter to prevent the valves from jamming. A tube extending from the valve is divided into two near the inner corners of the eyes.

Two springs fitted on the left side of the device push the syringe. When the electromagnetic valve opens, the spring pressure is released, and the water within the syringe flows into the region near the inner corners of the user's eyes (Figure 3 and 4). We employ this motor-free mechanism based on the lightness and quietness of the device.

One drop of water, with a liquid volume of approximately 0.02 $\mathrm{ml}$ to $0.05 \mathrm{ml}$ is produced when the electromagnetic valve is opened for approximately $300 \mathrm{~ms}$. This water drop volume is approximately equal to one drop of an emotional tear [15].

\subsection{Communication}

The flow of water can be remotely controlled via infrared communication with $38-\mathrm{kHz}$ modulation. When the device receives a message, the electromagnetic valve opens to allow water flow.
Valve is closed: water stops flowing. Valve is open: water flows.

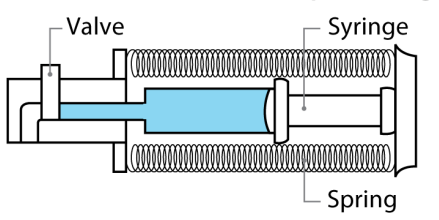

Figure 3: Mechanism of water flow. Two springs are always pushing the syringe. When the valve is closed, water stops flowing (left). When the valve is open, water flows (right).

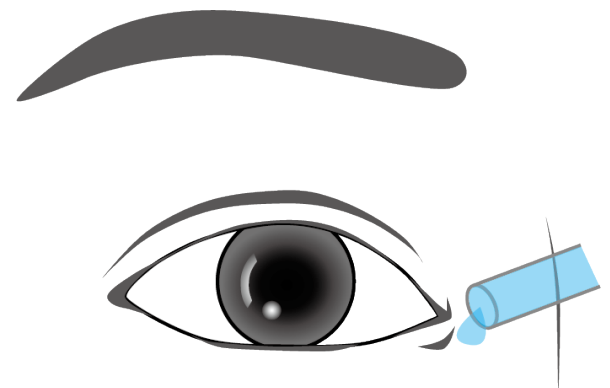

Figure 4: A drop of water flows onto the region near the inner corners of the wearer's eyes.

We developed a small, rectangular infrared communication module consisting primarily of an infrared LED and a photodiode positioned on opposite sides, along with a microcontroller (Arduino Pro Mini, $16 \mathrm{MHz}$ ). The size of the communication module was approximately $10 \mathrm{~mm} \times 16 \mathrm{~mm} \times 17 \mathrm{~mm}$ without the microcontroller. This module can be attached to the corners of a PC monitor. The infrared LED sends messages to the device based on the brightness measured by the photodiode. If the module detects the white color 


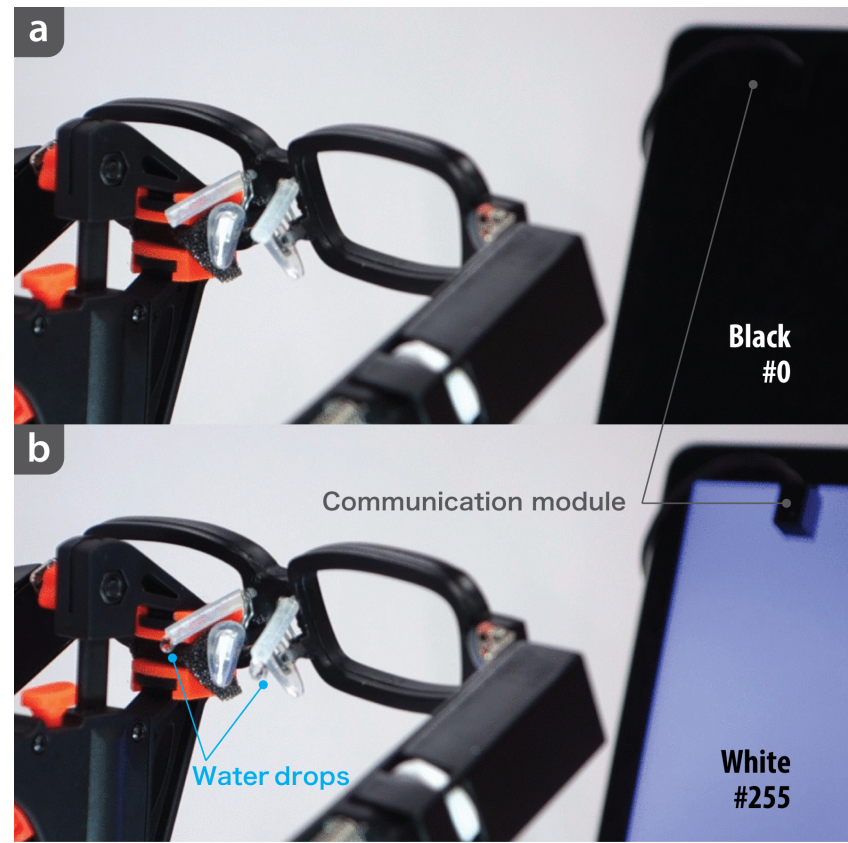

Figure 5: The communicatioin module, which is attached to the monitor, reads the brightness behind the module and control the flow of the water. (a) Water flow stops when the display brightness turns black. (b) Water flows when the display brightness turns white.

(\#255), it sends a message to the device to allow water flow. Meanwhile, if the module detects black color (\#0), it sends a message to stop the water flow (Figure 5). Hence, the control information of the eyeglasses can be embedded into a rim (only the part placed in the communicatioin module) of the content shown on the monitor. In addition, tears can only flow while the user is facing the monitor with the module because of the directional characteristic of the infrared LED.

\section{EVALUATION}

\subsection{Study Design}

In this study, we investigated whether artificial crying with pseudo tears, which are generated by our device, can influence emotions, especially sadness. The existence of explicit social interactions among participants causes ambiguity in the type of emotions that would be induced by artificial crying. As the first step of our investigation, we assumed a situation without social interactions. We measured the effect of artificial crying on the emotions of the participants while they were engaged in a passive activity that does not include social interactions with others, for instance, watching neutral visual content. Since tears typically connote sadness in the absence of apparent emotional contexts, we expected that the participants felt sadness even when they watched neutral visual content.

After each participant had received instructions regarding the study, we ensured that tears flowed near the inner corners of a participant's eyes and did not directly enter his/her eyes while using the device. The temperature of the water poured into the syringe was approximately $40^{\circ} \mathrm{C}$, which is higher than the body temperature, to account for the subsequent decrease in temperature before the water is released from the eyeglasses.

It should be noted that Mori et al. [45] showed that drops of water applied to the temple could not invoke sad feelings, in contrast to drops of water applied to the cheek. Therefore, in our tests, we only produced water droplets near the inner corners of the participants' eyes.

This study was approved by the local ethics committee.

\subsection{Visual Stimuli}

The participants were asked to watch three different 10 min slide shows. Each slide show consisted of 40 neutral images randomly selected from 168 neutral images in the International Affect Picture System (IAPS) database [42]. The emotional rating of the images as neutral is described in [21]. We used visual stimuli involving neutral images in this study to prevent the participants from inferring that this study aimed to measure sadness or other particular emotions. The order of the images was randomized for each participant group and condition, which is described later. Each image was presented for $15 \mathrm{~s}$ (40 images $\times 15 \mathrm{~s}=10 \mathrm{~min})$.

\subsection{Setup}

The slide shows were displayed using an LCD color monitor (EIZO FlexScan S2432W, 24.1 inches, $1600 \times 1000$ pixels) placed on a table in a closed quiet room. Each image was displayed in the center of a black background. The height of each image was 1000 pixels, and the width varied depending on the aspect ratio of each image. The monitor was positioned approximately $1 \mathrm{~m}$ in front of the participants. The stimulus presentation was controlled using a computer (Apple MacBook Pro Retina 15 inch Late 2013, 2.6 GHz Intel Core i7, 16 GB RAM, NVIDIA GeForce GT 750M, running OS $\mathrm{X}$ 10.11.3).

A rectangular color code (black or white) was embedded at the top left of each slide. The communication module was attached to that area with double-sided tape on the monitor to read the color code. The code color is typically black but turned white for a period of $300 \mathrm{~ms}$ in conjunction with the dropping of tears; subsequently, the code color returned to black. It should be noted that only the color behind the module was changed, and the other area was not changed. During each of these $300-\mathrm{ms}$ periods, the electromagnetic valve of the eyeglasses opened, and water flowed. The tear-dropping interval was determined based on a preliminary study, as described below.

The participants used noise-canceling earplugs to reduce audible noise while they were watching the visual stimuli during the study to ensure that they concentrated on the visual stimuli.

\subsection{Preliminary Study: Tear-dropping Interval}

We performed a preliminary study to determine the interval for dropping tears. To avoid degradation of the visual content viewing experiences as a result of the pseudo tears, the frequency of tears had to be within a range that did not interfere with watching the visual content. The participants were asked to watch four different 5-min slides that had approximately the same visual stimuli as described above, except that they consisted of half the number of 
images. Tear-dropping intervals of 50, 25, 10, and $5 \mathrm{~s}$ were tested. The order of the intervals was randomized for each participant.

After watching each slide show, participants were asked to rate their responses to the question "Were you able to focus on watching the slide show?” on a seven-point Likert scale (1 = strongly disagree, $7=$ strongly agree). We recruited four participants (three males and one female) for this study. They did not know the purpose of this research.

The average ratings and their standard errors for the intervals of $50,25,10$, and $5 \mathrm{~s}$ were $4.0 \pm 0.58,3.5 \pm 0.5,3.25 \pm 1.11$, and $1.5 \pm$ 0.29 , respectively. These values indicate that the shortest interval of tear dropping (5 s) interfered noticeably with the participants' concentration while watching the slide show. In contrast, the degrees of concentration maintained by the participants were similar for the tear-dropping intervals of 10, 25, and $50 \mathrm{~s}$.

We concluded that the participants might not have focused adequately on watching the slide shows if tears flowed down at short intervals. In contrast, a low frequency of tear flow might not influence the emotion of the people watching the slide shows. Thus, we determined that the water should be dropped every $25 \mathrm{~s}$, which was the second of the three intervals for which similar results were obtained.

\subsection{User Study: Emotional Contagion by Artificial Crying}

This study examined whether emotional contagion could be triggered by artificial crying with pseudo tears. Our main objective was to evaluate the feelings of the participants who observed the artificial crying. Although the effects of direct presentation of pseudo tears have already been investigated $[43,45]$, we also measured the feelings of the participants who had presented pseudo tears to confirm that the participants shared similar emotions.

In this study, three-participant groups watched three slide shows involving neutral images from IAPS [42] together under three different conditions. The control condition where none of the participants wore the glasses and only watched a slide show was used to measure the baseline of the participants' emotions. We compared the differences in the effects generated when the participants watched a slide show with pseudo tears and under the control condition. To compensate for the effect of simply wearing the glasses, the condition in which participants wore the Teardrop glasses but no water flowed down their cheeks was also used in this study. One participant (wearer) in a group wears the glasses in each condition except the control condition; a wearer and two non-wearers were determined based on the answers of a questionnaire collected at the beginning of the study.

4.5.1 Participants. Twenty-one participants (nine males and twelve females; mean age $=22.2$ years; standard deviation $=4.21$ ) participated in the study. None of the participants participated in the preliminary study. Each received an Amazon gift card worth approximately $\$ 19(¥ 2,000)$ for their participation.

Research have shown that the degree of emotional contagion of sadness differs depending on the interpersonal relationships of the people involved [37], in that emotional facial mimicry of a negative emotion is less likely to occur among strangers [5], and that the susceptibility to other people's feelings differs according to gender
[31]. Thus, we recruited participants in groups of three persons of the same gender, including friends, seniors, and juniors, who were already acquainted with each other.

A total of seven groups (three groups of males and four females) participated in this study. We did not inform the participants that the real purpose of the study was to measure the their emotions. Rather, we asked them to write down their impressions regarding each slide show. In addition, the participants were informed that the eyeglasses used in the study would reproduce the sensation of shedding tears, as the participants might have been surprised if they had no prior warning that water drops would be flowing down their faces.

Note that if the participants usually wore eyeglasses, we asked them to wear contact lenses instead. After the study, we explained the purpose of the experiment to those who wished to know.

4.5.2 Condition. This study used a within-participants design. The participants watched three slide shows of different conditions:

- Control: Participants watch a slide show without the glasses. (baseline of participants' emotions)

- Glasses-without-tears: Participants wear the glasses while watching a slide show, but no water drops fall.

- Glasses-with-tears: Participants wear the glasses and are given some tissues to wipe tears away while watching a slide show. The water drops every $25 \mathrm{~s}$. One or two drops of water fall down when the electromagnetic valve opens.

The order of the conditions was randomly assigned and counterbalanced across groups using a Latin-square design. The images used in each condition and their order were also randomized, but there were some cases in which the same images appeared in multiple conditions.

In the glasses-with-tears condition, we allowed the participants to wipe away tears with tissues because the wiping away tears is an emotional expression that accompanies crying naturally.

4.5.3 Procedure. The participants were asked to sit in front of a PC monitor and watch three different 10-min slide shows. Moreover, the participants were told not to speak with each other during the study to eliminate the effect of social interactions. Informed consent was obtained from each participant after the instructions about the study were provided.

Before watching the first slide show, the participants were asked to complete an assessment of their susceptibility to other persons' emotions using the Emotional Contagion Scale (ECS) [11]. The Japanese version of the ECS questionnaire was used [38]. The ECS questionnaire consists of 15 questions, and someone who is likely to mimic the emotions of the people around him/her presumably obtains a higher rating. To eliminate the weak influence of emotional contagion resulting from a deficiency in empathy, the participant who obtained the lowest rating in a given group was chosen to be the wearer, such that he/she could wear the eyeglasses during each condition except for the control condition while watching the slide show. The other two participants (non-wearers) in the same group did not wear the glasses while watching the slide show. However, they were also aware of the functionality of the glasses.

To cause non-wearers to easily notice that the wearer appeared to be crying during the study, the wearer sat at the center of the 
group. Non-wearers were allowed to see wearers while watching the slide shows.

After watching each slide show, we asked the participants to write down their impressions regarding each slide show, in addition to recording their emotional ratings using a self-report inventory. Then, the participants had a 5-min break to reset their feelings.

Following the three sessions under different conditions, the participants were asked to write what they thought of or noticed during the study. Finally, we gave the participants an Amazon gift card (\$19) preloaded with an amount slightly higher than the standard hourly fees to compensate for the negative feelings that might have been evoked by this study.

The study lasted at most $90 \mathrm{~min}$.

4.5.4 Hypotheses. Previous studies have shown the relationship between tears and sadness [20,45]. Moreover, according to Russell's circumplex model of emotion [54], happiness has an inverse relationship with sadness. This led to the following hypotheses:

- H1-a: The sadness of non-wearers in the glasses-with-tears condition will be higher than that in other conditions.

- H2-a: The happiness of the non-wearers in the glasses-withtears condition will be lower than that in other conditions.

In addition, we hypothesized that artificial crying changes participants' impressions of visual stimuli. We assumed that the texts that the participants wrote after the glasses-with-tears condition would include more negative phrases than those written after other conditions; for example, researchers $[22,23]$ found that the text messages written by the participants were influenced by their emotions. In this study, we asked the participants to describe three topics that relate to visual stimuli, such as their impressions of it, what they thought and remembered while watching them, and the scenes they remember. Three different topics were used because the participants might write only a few comments about each topic. From the above, the following hypothesis was considered:

- H3-a: The positive-negative valence of the text by the nonwearers under the glasses-with-tears condition will be lower than those under other conditions.

Finally, we assumed that the emotions of the wearers were also influenced by the pseudo tears and they felt like the non-wearers. The three hypotheses below apply to wearers:

- H1-b: The sadness of the wearers in the glasses-with-tears condition will be higher than those in other conditions.

- H2-b: The happiness of the wearers in the glasses-with-tears condition will be lower than those under other conditions.

- H3-b: The positive-negative valence of the text by the wearers under the glasses-with-tears condition will be lower than those under other conditions.

4.5.5 Measurements. We used a self-report inventory to measure participants' emotions in accordance with prior works on crying and emotional contagion [20,28]. The participants were asked to rate their emotions after watching each slide show. In this study, five feelings (love, anger, happiness, sadness, and shame) were rated on a nine-point Likert scale $(1=$ strongly disagree, 9 = strongly agree). However, our primary interests were feelings of sadness and happiness. Thus, other feelings were used to ambiguate the focus of the study to the participants.
To evaluate the positive-negative valence of the participants, we used the sentiment analysis of the Google Cloud Natural Language API ${ }^{1}$. This sentiment analysis can identify prevailing emotions from the text. The scores are normalized within -1.0 (negative) to 1.0 (positive). In this study, we analyzed the texts regarding the impressions of the slide shows obtained from the participants using this API.

\subsection{Results}

Figure 6 shows boxplots of sadness (left) and happiness (right), rating changes of the glasses-without-tears and the glasses-withtears conditions. Data were collected by subtracting the sadness and happiness ratings in the control condition (emotion ratings in the glasses without/with tears condition - emotion ratings in the control condition). A Wilcoxon signed-rank test was performed to evaluate the sadness and happiness changes among the wearers and non-wearers. Statistical differences were found in sadness in both wearers and non-wearers (wearers: $Z=-2.31, p<0.05, r=$ 0.62 ; non-wearers: $Z=-2.49, p<0.05, r=0.47$ ). However, statistical differences were found in happiness only in non-wearers (wearers: $Z=-1.25, p=0.25, r=0.33$; non-wearers: $Z=-2.44, p<0.05, r=$ $0.46)$.

Figure 7 shows the average positive-negative scores of the texts that the participants wrote after watching the slide shows. The analysis method we used outputs normalized data ( -1.0 to 1.0$)$; therefore, we did not use the score in the control condition as a baseline and compared three scores without any modification. The scores were analyzed using a $2 \times 3$ mixed-design ANOVA to clarify the relations between the wearer/non-wearers and the conditions. The analysis revealed that there was a significant interaction between the wearers/non-wearers and the conditions $(F(2,38)=3.74, p<0.05$, $\left.\eta^{2}=0.09\right)$. Multiple comparisons with the modified sequentially rejective Bonferroni revealed that the positive-negative scores in non-wearers under the glasses-with-tears condition were significantly lower than those of the control and glasses-without-tears condition $(p<0.05$, respectively).

\section{DISCUSSION}

\subsection{Emotional Effects of Artificial Crying}

The purpose of this study was to examine whether emotional contagion can be invoked using pseudo tears, and whether the participants share similar emotions. We expected the participants to feel sadder or less happy under the glasses-with-tears condition than under the other conditions. The results of the user study show that the sadness rating in the glasses-with-tears condition was higher than the ratings for the other conditions for both the wearers and non-wearers. This indicates that people around the wearer (non-wearers) are influenced by the artificial bodily changes of the wearer. Moreover, wearers also feel sadness by a droplet imitating a teardrop falling down the face. These results support H1-a and H1-b.

The happiness rating of non-wearers in the glasses-with-tears condition was lower than the ratings for the other conditions.

\footnotetext{
${ }^{1}$ https://cloud.google.com/natural-language
} 

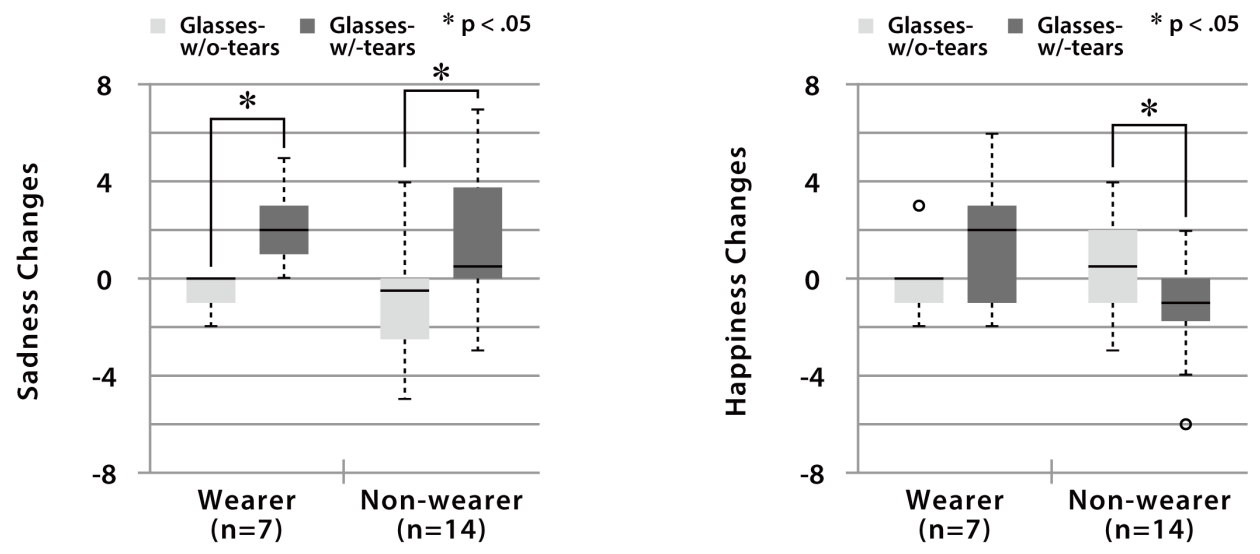

Figure 6: Boxplots showing the sadness (left) and happiness (right) changes of wearers and non-wearers. The changes are calculated by subtracting the ratings in the control condition from the ratings in other conditions (emotion ratings in the glasses-without/with-tears condition - emotion ratings in the control condition).

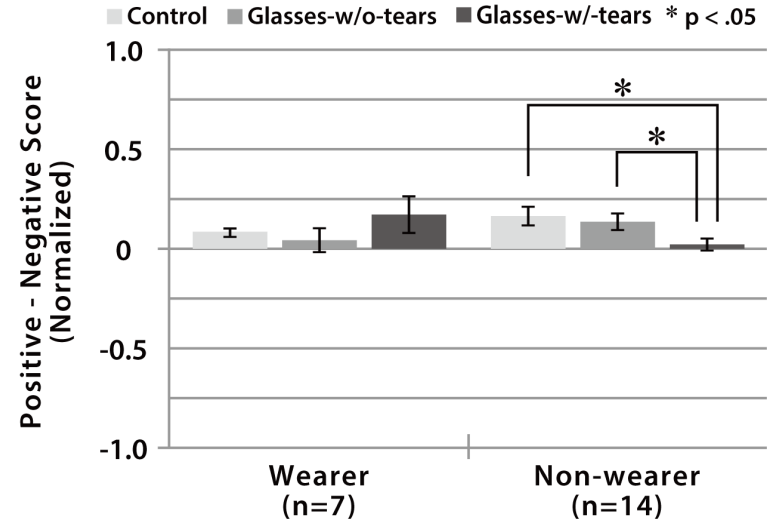

Figure 7: Average positive-negative scores estimated from the participants' texts written after each condition. Error bars denote standard errors.

However, no significant differences were found in the happiness ratings of the wearers. This supports $\mathrm{H} 2$-a but does not support $\mathrm{H} 2-\mathrm{b}$. Similarly, the positive-negative scores of the non-wearers in the glasses-with-tears condition were lower than those in the other conditions. No significant differences were found in the positivenegative scores of the wearers. This supports H3-a but does not support H3-b. These results suggest that the non-wearers are more influenced by artificial crying. This means that people who observed the artificial tears were more emotionally influenced than the people who shed the pseudo tears.

On the other hand, the participants might expect some relationship between sadness and teardrops produced by the device, regardless of the visual stimuli. In this study, it was difficult to deny the possibility that wearing the device itself caused a priming effect. However, if sadness was primed, then the average happiness rating of wearers should have decreased (which was not observed in our study). Therefore, we believe that the results indicate that it is difficult to say that there was a priming effect.

We gathered feedback from the participants after the study. Some wearers reported that artificial crying influenced their emotions.

"I felt sad because the (pseudo) tears made me think I was actually crying."

"I felt that the slide show in this session (glasses-with-tears condition) was sad compared to the slide show in the first session (control condition)."

Similarly, one non-wearer mentioned that artificial crying influenced his/her emotions:

"The person next to me wiping his/her tears made me sad even though the slide show was neutral."

One participant from the wearers remarked on the reality of the pseudo tears:

"I had a strange feeling when the tears rolled down."

No participants were actually reported crying. One participant, however, commented:

"My nose was runny because I felt as if I was crying."

Although notable behavioral changes such as actual crying were not found in our study, the comments from the participants suggest the emotional effects of artificial crying.

\subsection{Greater Emotional Impact on Non-wearers}

The results of the user study suggest that the wearers were more emotionally influenced than non-wearers by the artificial crying. In this study, to eliminate the weak influence of emotional contagion resulting from a deficiency in empathy, we used the ECS questionnaire to divide the participants into wearers and non-wearers. Since the ECS is used to measure susceptibility to other people's emotions, we assigned participants with low ECS scores as wearers who present pseudo tears as their own. This is because we assumed that there is no correlation between ECS scores and the degree to which emotions were elicited by perceiving the pseudo tears as their own bodily changes. However, people who are less affected by the emotions of others tend to show less emotional expressions [26]. This 
implies that individuals with low ECS scores are less susceptible to artificial bodily changes. This might cause differences in perceived emotions of pseudo tears.

In addition, the differences in the perceived reality of tears between wearers and non-wearers might be a factor that influenced their emotions. From the wearer's perspective, the device only provides a haptic sensation on the cheeks, which is not the complete sensory experience of crying. For non-wearers, there is no way to determine whether the tears were real or not, because the device effectively occluded the wearer's eyes, preventing non-wearers from seeing the full facial expression.

\subsection{Stress Relief Through Pseudo Tears}

The happiness rating and the average positive-negative score of wearers of the glasses-with-tears condition were higher than those in other conditions, although significant differences were not found. The feedback from the participants help to explain this. Some comments by the participants suggest that they associate pseudo tears with stress relief. One participant remarked that he/she felt refreshed by the pseudo tears:

"Finally, I felt refreshed and settled down."

Another participant remarked that he/she wanted to use the device for stress relief:

"I thought about using this device again when I want to relieve stress."

In fact, tears, typically considered an expression of distress, are believed to be involved in physiological or cognitive mechanisms for decreasing the stress reaction and causing someone to feel relaxed [19]. Such an effect might have a positive influence on the wearers in the glasses-with-tears condition. In this study, we focused solely on the emotional aspect of tears, but the participants' comments suggest the intriguing possibility that our device can partially reproduce the homeostatic regulation of tears. If this is true, this type of technology can be beneficial in the field of mental health and well-being and can be used to help people cope with stressful situations in their everyday lives.

None of the non-wearers, however, mentioned feeling refreshed after the pseudo tears, the results of the user study showing that they did not have higher positive emotions under the glasses-withtears condition. This means that the pseudo tears did not refresh the non-wearers, and only the emotional influence of tears appeared to be shared among the participants.

\subsection{Usage Scenarios}

In this study, we proposed Teardrop glasses to augment the viewing behavior and experiences emotionally through artificial crying. It is no longer unusual to wear glasses to augment viewing behaviors and experiences, such as wearing eyeglasses to supplement their vision and wearing 3D glasses to view stereoscopic images. Additionally, the form of glasses is desirable to present the pseudo tears naturally near the wearer's eyes because glasses are typically worn around the eyes. Thus, Teardrop glasses employs a simple tear-dropping mechanism that enables them to be as close as possible to actual glasses in size, lightness, and shape such that people can wear it as eyeglasses without discomfort.
Our findings can be applied to movie theaters and amusement parks to enhance the subjective reality through the direct inducement of emotions, as 3D glasses are commonly used in these places, and our mechanism can be installed easily on 3D glasses. Our findings suggest that, in these situations, pseudo-tearing capability does not need to be installed on all devices because emotional contagion can be used.

We found that our findings might be useful for promoting mental and social health in group therapy, regulating emotions of participants with emotional contagion triggered by a therapist's pseudo emotional expression. We expect that Teardrop glasses can be used to alleviate the symptoms caused by psychological factors including shedding tears, as mentioned earlier, have the effect of relieving their mood $[3,6]$.

Finally, we considered that the proposed device may be useful for releasing tears to evoke particular emotions. In the same manner a "laugh track" is commonly used in comedy drama, pseudo tears can be used as a "cry track" for inducing and enhancing sadness or an impressive situation, with tears as feedback, while watching movies at home. For the current implementation, the color code (the brightness of the display) was used to synchronize the timing for dropping tears and the elapsed time of the stimuli. The control module reads the brightness of the color code that is embedded in the slide show and sends the commands to the device. This simple control method allows not only programmers or engineers but also filmmakers and directors to freely design the timing of dropping tears by simply embedding the color code in the film they make, leading them to produce movies that can directly influence the emotional experiences of the viewers.

\section{LIMITATIONS AND FUTURE WORK}

\subsection{Other Emotions}

We also cry when we are happy or impressed by someone/something, as crying enhances our feelings in such situations. In future studies, it would be interesting to evaluate whether artificial crying can enhance emotional experiences while the participants are watching a happy or sad video, instead of a neutral video. We would like to evaluate whether significant differences exist depending on the type of video stimuli used. We believe that the use of other emotional video stimuli, such as those evoking happiness or sadness, increases the intensity of the emotion evoked and the variety of emotions.

\subsection{Wiping Tears Away}

The independent effects of pseudo tears and wiping tears were not revealed in this study. Since the objective of our study is to clarify "the effect of artificial crying" and not merely the teardrops, we decided to allow natural actions that accompanied shedding tears. Moreover, there was a possibility that the unnaturalness of not wiping away tears may induce a different emotion than what we intended. Therefore, we allowed the wearers to wipe away tears during the study.

\subsection{Hygiene Issues}

We did not pour water directly into the eyes of the participants because of hygiene considerations. However, if the hygiene issue 
can be adequately addressed, it would be possible to reproduce the sensation of gathering tears in the eyes and of the tears flowing through the lachrymal ducts, which causes a runny nose. This would improve the effect of the emotional evocation by truly reproducing the phenomenon: crying.

\subsection{Physiological Measurements}

In this study, we found that artificial tears influenced the subjective experiences of the participants. However, the behavioral changes related to crying were not found, such as actually shedding tears. Because the subjective results were confirmed through our study as the first step in exploring the concept of emotional contagion with artificial bodily changes, further research is required to investigate whether physiological changes can be observed through the measurement of physiological responses, such using electroencephalogram (EEG), facial electromyography (EMG), skin conductance, or heart rate responses.

\subsection{Sociocultural Difference}

To test whether artificial bodily changes induce emotional contagion, we gathered a group of participants in a close relationship to invoke emotional contagion effectively. Since emotional contagion is an innate nature of humans, we expect that the same phenomenon can be observed in a group of people with various relationships. However, further study is needed to demonstrate how emotional influence differs depending on the relationship between people within the group, as well as the group size.

On the other hand, some may not want to shed tears in public. However, we assume that shedding tears is acceptable for many people, depending on the situation described as usage scenarios, such as amusement, entertainment, and therapy. In addition, it should be noted that the benefit of our device is that if the user does not want to show they are shedding tears, they can simply remove the device.

\subsection{Design of Device}

Although none of the participants raised concerns regarding the current style of the device, we believe that we should consider alternative designs for those who wear headwear and eyeglasses.

In this study, we designed and implemented an eyeglasses-style wearable device. However, we believe that any style of the device that can drop water droplets around the eyes will reproduce the same effect.

Moreover, the bulky arms, from the exposed springs and syringe, and the battery, interfere with wearing the device for people who already have a headwear, such as a hat, headscarf, or turban. We used a syringe for sealing, ease of water replacement, and aspect of hygiene, but we believe that changing the device's structure to keep the water and spring inside the device make the arm more slender. Replacing a AAA battery with a smaller LiPo battery also contributes to reducing the arm.

\section{CONCLUSION}

We proposed a method for mediating people's emotions through emotional contagion using artificial bodily changes that can reproduce the sensation of crying. We developed an eyeglasses-style wearable device called Teardrop glasses that apply drops of water to the regions near the inner corners of the wearer's eyes at a controlled rate. A user study showed that artificial crying with pseudo tears caused the participants wearing the glasses to be sad and also caused other participants around them to be sad.

We believe that this is the first study to demonstrate that artificial crying can invoke sad feelings in both the participants who shed tears and their observers. We conclude from the results of this study that our minds can be mediated not only by pseudo changes to our own behavior but also by the observation of pseudo changes in other people's emotional expressions. Moreover, non-wearers were more emotionally influenced by artificial crying than wearers. Their happiness rating was significantly lower when pseudo tears were presented. The text analysis of their impressions of the visual contents also supports the emotional influence of artificial crying. These findings contribute to the design interactions between humans and their minds using technology.

\section{ACKNOWLEDGMENTS}

We would like to thank Michiteru Kitazaki, Daisuke Sakamoto, and Takafumi Oyaka for their insightful comments and discussions. This work was supported by JSPS KAKENHI Grant Numbers JP14J09015 and JP16K12471.

\section{REFERENCES}

[1] Shantha Amrith, Poh Sun Goh, and Shih-Chang Wang. 2005. Tear flow dynamics in the human nasolacrimal ducts?a pilot study using dynamic magnetic resonance imaging. Graefe's Archive for Clinical and Experimental Ophthalmology 243, 2 (2005), 127-131.

[2] Martijn JH Balsters, Emiel J Krahmer, Marc GJ Swerts, and Ad JJM Vingerhoets. 2013. Emotional tears facilitate the recognition of sadness and the perceived need for social support. Evolutionary Psychology 11, 1 (2013), 147470491301100114.

[3] Marleen C Becht and Ad JJM Vingerhoets. 2002. Crying and mood change: A cross-cultural study. Cognition \& Emotion 16, 1 (2002), 87-101.

[4] Timothy W. Bickmore and Rosalind W. Picard. 2005. Establishing and Maintaining Long-Term Human-Computer Relationships. ACM Trans. Comput.-Hum. Interact. 12, 2 (June 2005), 293-327. https://doi.org/10.1145/1067860.1067867

[5] Patrick Bourgeois and Ursula Hess. 2008. The impact of social context on mimicry. Biological psychology 77, 3 (2008), 343-352.

[6] Lauren M Bylsma, Ad JJM Vingerhoets, and Jonathan Rottenberg. 2008. When is crying cathartic? An international study. Journal of Social and Clinical Psychology 27,10 (2008), 1165-1187.

[7] Jean Costa, Alexander T. Adams, Malte F. Jung, François Guimbretière, and Tanzeem Choudhury. 2016. EmotionCheck: Leveraging Bodily Signals and False Feedback to Regulate Our Emotions. In Proceedings of the 2016 ACM International foint Conference on Pervasive and Ubiquitous Computing (Heidelberg, Germany) (UbiComp '16). Association for Computing Machinery, New York, NY, USA, 758-769. https://doi.org/10.1145/2971648.2971752

[8] Jean Costa, Malte F. Jung, Mary Czerwinski, François Guimbretière, Trinh Le, and Tanzeem Choudhury. 2018. Regulating Feelings During Interpersonal Conflicts by Changing Voice Self-Perception. In Proceedings of the $2018 \mathrm{CHI}$ Conference on Human Factors in Computing Systems (Montreal QC, Canada) (CHI '18). Association for Computing Machinery, New York, NY, USA, 1-13. https://doi.org/10.1145/3173574.3174205

[9] Antonio R Damásio. 1994. Descartes' error: emotion, reason, and the human brain. Quill, New York, NY, USA.

[10] Ulf Dimberg and Monika Thunberg. 2012. Empathy, emotional contagion, and rapid facial reactions to angry and happy facial expressions. PsyCh fournal 1, 2 (2012), 118-127.

[11] R William Doherty. 1997. The emotional contagion scale: A measure of individual differences. Fournal of nonverbal Behavior 21, 2 (1997), 131-154.

[12] R Linsy Farris. 1985. Tear analysis in contact lens wearers. Transactions of the American Ophthalmological Society 83 (1985), 501.

[13] Emilio Ferrara and Zeyao Yang. 2015. Measuring emotional contagion in social media. Plos one 10, 11 (2015), e0142390.

[14] William H Frey, Denise Desota-Johnson, Carrie Hoffman, and John T McCall. 1981. Effect of stimulus on the chemical composition of human tears. American journal of ophthalmology 92, 4 (1981), 559-567. 
[15] William. H. Frey and M. Langseth. 1985. Crying: the mystery of tears. Winston Press.

[16] Shogo Fukushima and Hiroyuki Kajimoto. 2012. Facilitating a Surprised Feeling by Artificial Control of Piloerection on the Forearm. In Proceedings of the $3 r d$ Augmented Human International Conference (Megève, France) (AH '12). Association for Computing Machinery, New York, NY, USA, Article 8, 4 pages. https://doi.org/10.1145/2160125.2160133

[17] Tom Gayler, Corina Sas, and Vaiva Kalnikaite. 2019. Taste Your Emotions: An Exploration of the Relationship between Taste and Emotional Experience for HCI. In Proceedings of the 2019 on Designing Interactive Systems Conference (San Diego, CA, USA) (DIS '19). Association for Computing Machinery, New York, NY, USA, 1279-1291. https://doi.org/10.1145/3322276.3322336

[18] Takashi Goto, Benjamin Tag, Kai Kunze, and Tilman Dingler. 2018. Towards En hancing Emotional Responses to Media Using Auto-Calibrating Electric Muscle Stimulation (EMS). In Proceedings of the 9th Augmented Human International Conference (Seoul, Republic of Korea) (AH '18). Association for Computing Machinery, New York, NY, USA, Article 29, 2 pages. https://doi.org/10.1145/3174910.3174939

[19] Asmir Gračanin, Lauren M Bylsma, and Ad JJM Vingerhoets. 2014. Is crying a self-soothing behavior? Frontiers in Psychology 5 (2014).

[20] James J Gross, Barbara L Fredrickson, and Robert W Levenson. 1994. The psychophysiology of crying. Psychophysiology 31, 5 (1994), 460-468.

[21] Daniel Grühn, Susanne Scheibe, and Paul B Baltes. 2007. Reduced negativity effect in older adults' memory for emotional pictures: the heterogeneity-homogeneity list paradigm. Psychology and aging 22, 3 (2007), 644 .

[22] Jamie Guillory, Jason Spiegel, Molly Drislane, Benjamin Weiss, Walter Donner and Jeffrey Hancock. 2011. Upset Now? Emotion Contagion in Distributed Groups. In Proceedings of the SIGCHI Conference on Human Factors in Computing Systems (Vancouver, BC, Canada) (CHI '11). Association for Computing Machinery, New York, NY, USA, 745-748. https://doi.org/10.1145/1978942.1979049

[23] Jeffrey T. Hancock, Kailyn Gee, Kevin Ciaccio, and Jennifer Mae-Hwah Lin. 2008 I'm Sad You're Sad: Emotional Contagion in CMC. In Proceedings of the 2008 ACM Conference on Computer Supported Cooperative Work (San Diego, CA, USA) (CSCW'08). Association for Computing Machinery, New York, NY, USA, 295-298. https://doi.org/10.1145/1460563.1460611

[24] Mariam Hassib, Max Pfeiffer, Stefan Schneegass, Michael Rohs, and Florian Alt 2017. Emotion Actuator: Embodied Emotional Feedback through Electroencephalography and Electrical Muscle Stimulation. In Proceedings of the $2017 \mathrm{CHI}$ Conference on Human Factors in Computing Systems (Denver, Colorado, USA) (CHI '17). Association for Computing Machinery, New York, NY, USA, 6133-6146. https://doi.org/10.1145/3025453.3025953

[25] Oren Hasson. 2009. Emotional tears as biological signals. Evolutionary Psychology 7, 3 (2009), 147470490900700302.

[26] Elaine Hatfield, John T Cacioppo, and Richard L Rapson. 1992. Primitive emotional contagion. Review of personality and social psychology 14 (1992), 151-177.

[27] Elaine Hatfield, John T Cacioppo, and Richard L Rapson. 1993. Emotional contagion. Current directions in psychological science 2, 3 (1993), 96-100.

[28] Michelle CP Hendriks and Ad JJM Vingerhoets. 2006. Social messages of crying faces: Their influence on anticipated person perception, emotions and behavioural responses. Cognition and Emotion 20, 6 (2006), 878-886.

[29] Ursula Hess and Sylvie Blairy. 2001. Facial mimicry and emotional contagion to dynamic emotional facial expressions and their influence on decoding accuracy. International journal of psychophysiology 40, 2 (2001), 129-141.

[30] Paul Hill and Randall B Martin. 1997. Empathic weeping, social communication, and cognitive dissonance. Fournal of Social and Clinical Psychology 16, 3 (1997), $299-322$.

[31] Martin L Hoffman. 1977. Sex differences in empathy and related behaviors Psychological bulletin 84, 4 (1977), 712

[32] Mohammed (Ehsan) Hoque, Matthieu Courgeon, Jean-Claude Martin, Bilge Mutlu, and Rosalind W. Picard. 2013. MACH: My Automated Conversation Coach. In Proceedings of the 2013 ACM International foint Conference on Pervasive and Ubiquitous Computing (Zurich, Switzerland) (UbiComp '13). Association for Computing Machinery, New York, NY, USA, 697-706. https: //doi.org/10.1145/2493432.2493502

[33] Ken Iwasaki, Takashi Miyaki, and Jun Rekimoto. 2010. AffectPhone: A Handset Device to Present User's Emotional State with Warmth/Coolness.. In B-Interface. 83-88.

[34] William James. 1950. The principles of psychology. Dover Publications, New York, NY, USA.

[35] William James. 1994. The Physical Basis of Emotion. Psychological Review 101, (1994), 205-210. https://doi.org/10.1037/0033-295X.101.2.205

[36] Rohit Ashok Khot and Jung-Ying (Lois) Yi. 2020. GustaCine: Towards Designing a Gustatory Cinematic Experience. In Proceedings of the Fourteenth International Conference on Tangible, Embedded, and Embodied Interaction (Sydney NSW, Australia) (TEI '20). Association for Computing Machinery, New York, NY, USA, 757-770. https://doi.org/10.1145/3374920.3375010

[37] Masanori Kimura, Ikuo Daibo, and Masao Yogo. 2008. The study of emotional contagion from the perspective of interpersonal relationships. Social Behavior and Personality: an international journal 36, 1 (2008), 27-42.
[38] Masanori Kimura, Masao Yogo, and Ikuo Daibo. 2007. Development of a Japanese version of the Emotional Contagion Scale. Fapanese fournal of Interpersonal and Social Psychology 7 (2007), 31-39. In Japanese.

[39] Chris L Kleinke, Thomas R Peterson, and Thomas R Rutledge. 1998. Effects of self-generated facial expressions on mood. Fournal of Personality and Social Psychology 74, 1 (1998), 272.

[40] Adam D.I. Kramer. 2012. The Spread of Emotion via Facebook. In Proceedings of the SIGCHI Conference on Human Factors in Computing Systems (Austin, Texas, USA) (CHI '12). Association for Computing Machinery, New York, NY, USA, 767-770. https://doi.org/10.1145/2207676.2207787

[41] Adam DI Kramer, Jamie E Guillory, and Jeffrey T Hancock. 2014. Experimental evidence of massive-scale emotional contagion through social networks. Proceedings of the National Academy of Sciences 111, 24 (2014), 8788-8790.

[42] Peter J Lang. 2005. International affective picture system (IAPS): Affective ratings of pictures and instruction manual. Technical report (2005).

[43] Yu Liang, Kazuma Shimokawa, Shigeo Yoshida, and Eriko Sugimori. 2020. What "Tears" Remind Us of: An Investigation of Embodied Cognition and Schizotypal Personality Trait Using Pencil and Teardrop Glasses. Frontiers in Psychology 10 (2020), 2826. https://doi.org/10.3389/fpsyg.2019.02826

[44] Maria Miceli and Cristiano Castelfranchi. 2003. Crying: Discussing its basic reasons and uses. New ideas in Psychology 21, 3 (2003), 247-273.

[45] Hideko Mori and Kazuo Mori. 2007. A test of the passive facial feedback hypothesis: we feel sorry because we cry. Perceptual and motor skills 105, 3_suppl (2007), $1242-1244$

[46] J Murube, L Murube, and A Murube. 1999. Origin and types of emotional tearing. European fournal of Ophthalmology 9, 2 (1999), 77-84.

[47] Naoto Nakazato, Shigeo Yoshida, Sho Sakurai, Takuji Narumi, Tomohiro Tanikawa, and Michitaka Hirose. 2014. Smart Face: Enhancing Creativity during Video Conferences Using Real-Time Facial Deformation. In Proceedings of the 17th ACM Conference on Computer Supported Cooperative Work \& Social Computing (Baltimore, Maryland, USA) (CSCW '14). Association for Computing Machinery, New York, NY, USA, 75-83. https://doi.org/10.1145/2531602.2531637

[48] Roland Neumann and Fritz Strack. 2000. " Mood contagion": the automatic transfer of mood between persons. Fournal of personality and social psychology 79, 2 (2000), 211.

[49] Narihiro Nishimura, Asuka Ishi, Michi Sato, Shogo Fukushima, and Hiroyuki Kajimoto. 2012. Facilitation of Affection by Tactile Feedback of False Heratbeat. In CHI '12 Extended Abstracts on Human Factors in Computing Systems (Austin, Texas, USA) (CHI EA '12). Association for Computing Machinery, New York, NY, USA, 2321-2326. https://doi.org/10.1145/2212776.2223796

[50] Marianna Obrist, Sriram Subramanian, Elia Gatti, Benjamin Long, and Thomas Carter. 2015. Emotions Mediated Through Mid-Air Haptics. In Proceedings of the 33rd Annual ACM Conference on Human Factors in Computing Systems (Seoul, Republic of Korea) (CHI '15). Association for Computing Machinery, New York, NY, USA, 2053-2062. https://doi.org/10.1145/2702123.2702361

[51] Rosalind W. Picard. 1997. Affective Computing. MIT Press, Cambridge, MA, USA.

[52] Rosalind W. Picard, Elias Vyzas, and Jennifer Healey. 2001. Toward Machine Emotional Intelligence: Analysis of Affective Physiological State. IEEE Trans. Pattern Anal. Mach. Intell. 23, 10 (Oct. 2001), 1175-1191. https://doi.org/10.1109/ 34.954607

[53] Robert R Provine, Kurt A Krosnowski, and Nicole W Brocato. 2009. Tearing: Breakthrough in human emotional signaling. Evolutionary Psychology 7, 1 (2009), 147470490900700107

[54] James A Russell and James M Carroll. 1999. On the bipolarity of positive and negative affect. Psychological bulletin 125, 1 (1999), 3.

[55] Yuka Sato and Ryoko Ueoka. 2017. Investigating Haptic Perception of and Physiological Responses to Air Vortex Rings on a User's Cheek. In Proceedings of the 2017 CHI Conference on Human Factors in Computing Systems (Denver, Colorado, USA) (CHI '17). Association for Computing Machinery, New York, NY, USA, 3083-3094. https://doi.org/10.1145/3025453.3025501

[56] Stanley Schachter and Jerome Singer. 1962. Cognitive, social, and physiological determinants of emotional state. Psychological review 69, 5 (1962), 379.

[57] Norbert Schwarz. 2011. Feelings-as-information theory. Handbook of theories of social psychology 1 (2011), 289-308.

[58] Fritz Strack, Leonard L Martin, and Sabine Stepper. 1988. Inhibiting and facilitating conditions of the human smile: a nonobtrusive test of the facial feedback hypothesis. Fournal of personality and social psychology 54, 5 (1988), 768.

[59] Keita Suzuki, Masanori Yokoyama, Shigeo Yoshida, Takayoshi Mochizuki, Tomohiro Yamada, Takuji Narumi, Tomohiro Tanikawa, and Michitaka Hirose. 2017. FaceShare: Mirroring with Pseudo-Smile Enriches Video Chat Communications. In Proceedings of the 2017 CHI Conference on Human Factors in Computing Systems (Denver, Colorado, USA) (CHI '17). Association for Computing Machinery, New York, NY, USA, 5313-5317. https://doi.org/10.1145/3025453.3025574

[60] Hitomi Tsujita and Jun Rekimoto. 2011. Smiling Makes Us Happier: Enhancing Positive Mood and Communication with Smile-Encouraging Digital Appliances. In Proceedings of the 13th International Conference on Ubiquitous Computing (Beijing, China) (UbiComp '11). Association for Computing Machinery, New York, NY, USA, 1-10. https://doi.org/10.1145/2030112.2030114 
[61] Stuart Valins. 1966. Cognitive effects of false heart-rate feedback. Fournal of personality and social psychology 4, 4 (1966), 400.

[62] Shigeo Yoshida, Tomohiro Tanikawa, Sho Sakurai, Michitaka Hirose, and Takuji Narumi. 2013. Manipulation of an Emotional Experience by Real-Time Deformed
Facial Feedback. In Proceedings of the 4th Augmented Human International Conference (Stuttgart, Germany) ( $A H$ '13). Association for Computing Machinery, New York, NY, USA, 35-42. https://doi.org/10.1145/2459236.2459243

[63] Heng Zhu and Anuj Chauhan. 2005. A mathematical model for tear drainage through the canaliculi. Current eye research 30, 8 (2005), 621-630. 